\title{
Momentum bandwidth of the KEK Accelerator Test Facility 2
}

\author{
R. Yang $\odot,{ }^{1, * \dagger}$ A. Pastushenko $\odot,{ }^{1,4}$ V. Cilento $\odot,{ }^{1,4}$ K. Kubo, ${ }^{2,3}$ T. Naito, ${ }^{2,3}$ T. Okugi, ${ }^{2,3}$ \\ N. Terunuma, ${ }^{2,3}$ and R. Tomás ${ }^{1}$ \\ ${ }^{1}$ European Organization for Nuclear Research, Geneva CH-1211, Switzerland \\ ${ }^{2}$ High Energy Accelerator Research Organization, Tsukuba, Ibaraki 305-0801, Japan \\ ${ }^{3}$ School of High Energy Accelerator Science, SOKENDAI, Tsukuba, Ibaraki 305-0801, Japan \\ ${ }^{4}$ IJCLab, CNRS/IN2P3, Université Paris-Saclay, Orsay 91898, France
}

(Received 20 January 2021; accepted 4 May 2021; published 21 May 2021)

\begin{abstract}
Momentum bandwidth is an important characteristic for final focus systems (FFS) of future high-energy colliders. A large momentum bandwidth is a notable feature of the novel local-chromaticity-correction FFS, being demonstrated at the KEK Accelerator Test Facility 2 (ATF2), owing to the locality of chromaticity correction. In this article, analytical estimations and simulations of momentum bandwidth for various optics of ATF2 are presented. Possible deviations of the momentum bandwidth due to realistic machine imperfections are studied. Experimental measurements at ATF2 following successful tuning of small vertical beam size at the interaction point (IP) are also described, which agree well with numerical predictions and further verify the robustness of the local chromaticity correction scheme.
\end{abstract}

DOI: 10.1103/PhysRevAccelBeams.24.051001

\section{INTRODUCTION}

Delivery of ultrahigh luminosity for precise physics experiments with extremely small cross sections is one of the most critical challenges for the future linear collider (FLC). For this, particle beams have to be focused to the nanometer scale at the IP through the final focus system, which must be chromatically corrected due to the finite momentum spread $(0.1 \%-1 \%)$. There are mainly two types of final focus systems (FFS), traditional and local schemes, based upon nonlocal and local chromaticity corrections, respectively [1-3]. For the traditional scheme, paired sextupoles with $-I$ transformation in between are placed in dedicated horizontal and vertical chromaticity correction sections. The local scheme compensates chromaticity locally using two sextupoles interleaved with the final doublet (FD) and a bend upstream to generate the required dispersion. Thanks to the merit on reducing the total length of the FFS, especially in the multi-TeV energy scale [4], the local scheme has been considered for the latest baseline designs of the International Linear Collider (ILC) $[5,6]$ and the Compact Linear Collider (CLIC) [7,8].

\footnotetext{
renjun.yang@kek.jp

Present address: High Energy Accelerator Research Organization, Tsukuba, Ibaraki 305-0801, Japan.

Published by the American Physical Society under the terms of the Creative Commons Attribution 4.0 International license. Further distribution of this work must maintain attribution to the author(s) and the published article's title, journal citation, and DOI.
}

The momentum bandwidth is a vital parameter for the FFS designs. A wide and flat momentum bandwidth is always desirable for minimizing luminosity degradation by marginal beam-energy errors. The momentum bandwidth relates to the chromatic properties of FFS, e.g., chromaticity, second-order dispersion, and higher-order chromatic terms. For the traditional scheme, the momentum bandwidth might be dominated by the higher-order chromatic aberrations arising from the breakdown of $-I$ transformation between paired sextupoles [9]. Due to the locality of chromaticity correction, the local scheme's momentum bandwidth can be naturally wide without inserting additional nonlinear elements $[2,10]$. To acquire satisfactory momentum bandwidth, several optimization strategies have been developed, for instance, introduction of additional sextupoles (Brinkmann's sextupoles) and unequal dispersion at paired sextupoles ("odd-dispersion" scheme) $[4,11-13]$. In the reported literature $[1-4,14]$, the definition of momentum bandwidth of FFS is somewhat ambiguous. In this paper, we use momentum bandwidth to describe the chromatic proprieties of colliding beams in terms of IP $\beta$ function $\left(\beta^{*}\right)$ and beam size $\left(\sigma^{*}\right)$, in the units of onmomentum values. Moreover, the $10 \%$ momentum bandwidth $\left(\chi_{m}\right)$ is defined as a $10 \%$ increase of either horizontal or vertical IP spot size for mono-energetic beam. On the other hand, bandwidth with respect to luminosity performance has been used for CLIC FFS optimizations $[4,14,15]$.

Realistic machine imperfections can disrupt momentum bandwidth, as well as chromaticity compensation. Therefore, measurement of momentum bandwidth is crucial for 


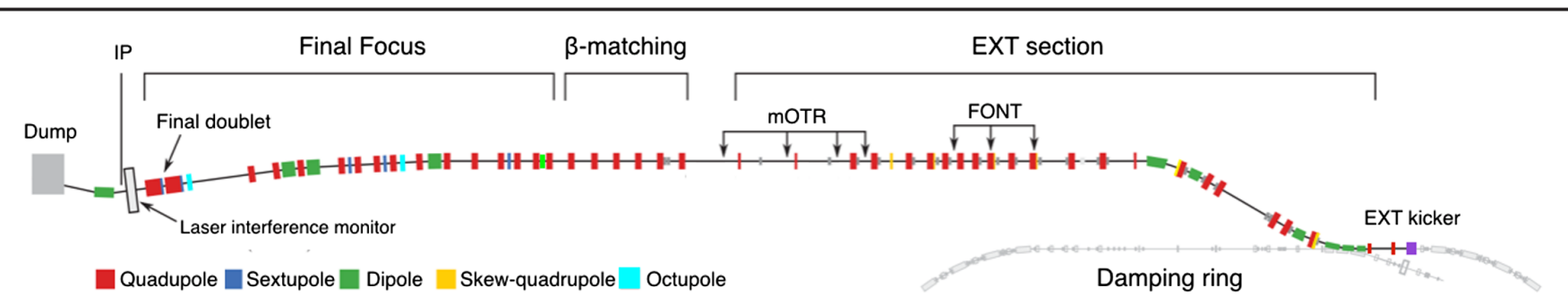

FIG. 1. Schematic diagram of the ATF2 beam line. mOTR: multi-optical transition radiation monitor; FONT: feedback on nanossecond timescale; EXT: extraction.

verifying the robustness of chromaticity correction, and providing insights into the residual high-order chromatic terms that might limit the IP spot size. Furthermore, the experimental observations are potentially useful for improving the reliability of FLC designs. For comparison with design optics, momentum-bandwidth measurement with a small IP beam size approaching design is favored, which requires enormous effort on beam tuning. At the final focus test beam (FFTB), a prototype FFS based on traditional scheme, the chromatic vertical waist shift and behavior of IP vertical spot size versus momentum spread have been measured [16-19]. As a test facility to demonstrate the local-chromaticity-correction scheme, the KEK-ATF2 provides a unique opportunity to investigate the momentum bandwidth of a local-style FFS, following the successful achievements of small IP vertical beam size below $60 \mathrm{~nm}$ [20-27]. In this report, $\beta$ function momentum bandwidth for a simplified analytical FFS model is described, followed by numerical simulations of ATF2 momentum bandwidths in the presence of realistic machine parameters. Measurements conducted for various ATF2 optics are then presented, which are consistent with numerical predictions.

\section{A. ATF2 beam line}

ATF2 has been constructed to demonstrate the local chromaticity correction scheme, provide a testbed for the development of advanced instrumentation, train the nextgeneration accelerator physicists and promote international collaboration toward building and operating FLC. The primary goal of ATF2 is to achieve a nanometer beam size with beam orbit stabilization in nanometer precision in the vertical plane at the IP. ATF2 mainly consists of the extraction section for beam extraction from the ATF damping ring (DR), the $\beta$-matching section and the FFS (see Fig. 1). The nominal ATF2 optics closely resembles the ILC with a target IP beam size of $37 \mathrm{~nm}$. To relax the sensitivity of IP vertical beam size to the magnetic multipolar aberrations, the $10 \beta_{x}^{*} 1 \beta_{y}^{*}$ optics with ten times larger IP horizontal $\beta$ function than the original design has been recently used $[28,29]$. Moreover, the ultralow $\beta^{*}$ optics $\left(25 \beta_{x}^{*} 0.25 \beta_{y}^{*}\right)$ has been tuned for demonstrating the tightest focusing possibility with a higher chromaticity beyond ILC and approaching CLIC [26,30-32]. The major parameters of the optics being studied at ATF2 are summarized in Table I. To measure nanometer scaled beam size, a laser interferometer beam size monitor (IPBSM), which has three crossing angles, $2-8^{\circ}, 30^{\circ}$ and $174^{\circ}$, to cover beam sizes from few micrometers to about $20 \mathrm{~nm}$, has been installed at the virtual IP [33-35].

\section{ANALYTIC APPROXIMATION}

To illustrate the basic properties of momentum bandwidth, analytical approximations have been derived concerning a simplified local-style FFS structure, as depicted in Fig. 2. This simple FFS model contains one sextupole adjacent to the final quadrupole for chromaticity correction, one paired sextupole upstream for correcting chromaticity and geometrical aberrations, and two dipoles for generating required horizontal dispersion. The bend upstream of the paired sextupole is introduced to reduce the chromaticity through the system. The derivation below is an extension to the analysis in Ref. [36], that is originally developed for traditional-style FFS. For sake of simplicity, the beam parameters at the entrance are assumed to be constant, and the chromaticity from upstream and chromatic aberrations are omitted. For an off-momentum particle, the transfer matrix from the entrance of sextupole at $s_{0}$ to the IP can be expressed as

$$
M=M_{L} M_{q} M_{s_{1}} M\left(s_{1} \mid s_{0}\right) M_{s_{0}}
$$

where $M_{L}, M_{q}, M_{s 1}$, and $M_{s_{0}}$ are the transfer matrices of the drift from the final quadrupole to the IP, the final quadrupole, the sextupoles at $s_{1}$ and $s_{0}$, respectively, and

TABLE I. Key FFS parameters of ATF2 various optics. $L^{*}$ is the distance between the last quadrupole and the IP, $\xi_{y}$ is the vertical chromaticity and $\sigma_{y}^{*}$ is the IP vertical beam size.

\begin{tabular}{lccc}
\hline \hline Optics & Nominal & $10 \beta_{x}^{*} 1 \beta_{y}^{*}$ & Ultralow $\beta^{*}$ \\
\hline$\beta_{x}^{*}[\mathrm{~mm}]$ & 4 & 40 & 100 \\
$\beta_{y}^{*}[\mu \mathrm{m}]$ & 100 & 100 & 25 \\
$L^{*}[\mathrm{~m}]$ & 1 & 1 & 1 \\
$\xi_{y} \sim L^{*} / \beta_{y}^{*}$ & $1 \times 10^{4}$ & $1 \times 10^{4}$ & $4 \times 10^{4}$ \\
$\sigma_{y, \text { design }}^{*}[\mathrm{~nm}]$ & 37 & 37 & 23 \\
$\sigma_{y, \text { measured }}^{*}[\mathrm{~nm}]$ & $\ldots$ & $42.3 \pm 2.7 / 41.1 \pm 0.7^{\mathrm{a}}$ & $50.1 \pm 0.6$ \\
\hline \hline
\end{tabular}

${ }^{\mathrm{a}}$ Results achieved with beam stabilization in two-bunch mode. 


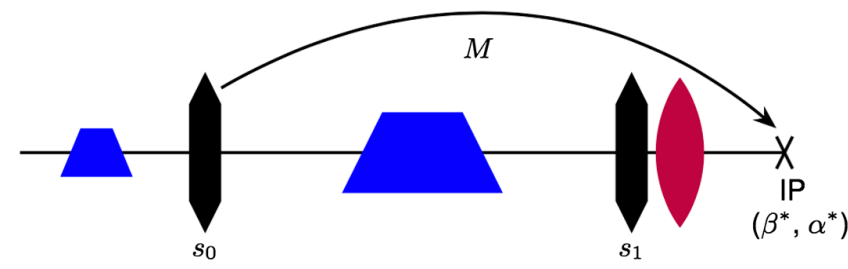

FIG. 2. Layout of a simplified FFS based on the localchromaticity-correction principle. Quadrupole magnet are shown in red, sextupoles in black, and dipoles in blue. The $s_{0}$ and $s_{1}$ label the sextupoles' locations.

$M\left(s_{1} \mid s_{0}\right)$ is the transfer matrix from the exit of the sextupole at $s_{0}$ to the entrance of the sextupole at $s_{1}$. Here, we ignore the gap between the final quadrupole and the neighboring sextupole. With thin-lens approximation, the transfer matrices of the final quadrupole and the sextupoles could be written as

$$
\begin{aligned}
M_{q} & =\left(\begin{array}{cc}
1 & 0 \\
-(1-\delta) K_{q} & 1
\end{array}\right) \\
M_{s_{0}} & =\left(\begin{array}{cc}
1 & 0 \\
-\delta K_{s_{0}} \eta_{x, s_{0}} & 1
\end{array}\right) \\
M_{s_{1}} & =\left(\begin{array}{cc}
1 & 0 \\
-\delta K_{s_{1}} \eta_{x, s_{1}} & 1
\end{array}\right)
\end{aligned}
$$

where $\delta=\Delta p / p$ denotes the relative momentum deviation, $K_{q}$ the quadrupole strength, $K_{s_{0}}$ and $K_{s_{1}}$ the integrated sextupole strengths, $\eta_{x, s_{0}}$ and $\eta_{x, s_{1}}$ the horizontal dispersions at the $s_{0}$ and $s_{1}$, respectively. For simplicity, we assume $M\left(s_{1} \mid s_{0}\right)$ as constant and infer it from the onmomentum transfer matrix $M$ with beam focused at the IP $\left(\alpha^{*}=0\right)$. The off-momentum IP $\beta$ function can then be given by

$$
\begin{aligned}
\frac{\beta^{*}(\delta)}{\beta_{0}^{*}}= & \frac{1}{\beta_{0}^{*}}\left(M_{11}^{2} \beta_{s_{0}}-2 M_{11} M_{12} \alpha_{s_{0}}+M_{12}^{2} \gamma_{s_{0}}\right) \\
= & \left\{-K_{s_{0}} \eta_{x, s_{0}} \beta_{s_{0}} K_{q}^{\prime} L^{*} \delta^{2}(\sin \psi-\xi \cos \psi)\right. \\
& +\left[K_{q}^{\prime} L^{*}(\cos \psi+\xi \sin \psi)-K_{s_{0}} \eta_{x, s_{0}} \beta_{s_{0}} \sin \psi\right] \delta \\
& +\cos \psi\}^{2}+\left[\sin \psi+K_{q}^{\prime} L^{*} \delta(\sin \psi-\xi \cos \psi)\right]^{2}(3)
\end{aligned}
$$

with

$$
K_{q}^{\prime}=K_{q}-K_{s_{1}} \eta_{x, s_{1}}
$$

where $\beta_{0}^{*}$ is the on-momentum IP $\beta$ function, $\beta_{s_{0}}, \alpha_{s_{0}}$ and $\gamma_{s_{0}}$ the Twiss parameters at $s_{0}, \xi=L^{*} / \beta^{*}$ the chromaticity generated by the strong final quadrupole, $L^{*}$ the distance from the last quadrupole to the IP and $\psi$ the phase advance between $s_{0}$ and IP. The expression of off-momentum IP $\beta$ function is quartic, and a region with $\beta^{*}(\delta)<\beta_{0}^{*}$ might exist if the chromatic effect is not completely canceled.

For the cancellation of the chromaticity and geometric aberrations, the phase advance $\psi$ is typically set at nearly $\left(n+\frac{1}{2}\right) \pi$, where $n$ is an integer, and $M_{12}^{s_{0} \rightarrow s_{1}}$ is optimized to be zero [2,36], i.e., $\sin \psi=\xi \cos \psi$. Subsequently, we can reduce Eq. (3) to a quadratic function, as

$$
\begin{aligned}
\frac{\beta^{*}(\delta)}{\beta_{0}^{*}} & =\left\{\left[\left(K_{q}^{\prime} L^{*} \delta\left(\xi+\frac{1}{\xi}\right)-K_{s_{0}} \eta_{x, s_{0}} \beta_{s_{0}} \delta+\frac{1}{\xi}\right]^{2}+1\right\} \sin ^{2} \psi\right. \\
& \approx\left\{\left[\left(K_{q}^{\prime} L^{*} \xi-K_{s_{0}} \eta_{x, s_{0}} \beta_{s_{0}}\right) \delta+\frac{1}{\xi}\right]^{2}+1\right\} \sin ^{2} \psi
\end{aligned}
$$

by assuming $\xi \gg \xi^{-1}$. Apparently, the momentum dependence of $\beta^{*}$ vanishes for $K_{q}^{\prime} L^{*} \xi=K_{s_{0}} \eta_{x, s_{0}} \beta_{s_{0}}$. Seeing that $\beta_{s_{0}}$ is roughly proportional to $1 / \beta^{*}$, the above equation can be further simplified to

$$
\frac{\beta^{*}(\delta)}{\beta_{0}^{*}} \approx\left[\left(\frac{\delta \zeta}{\beta_{0}^{*}}+\frac{1}{\xi}\right)^{2}+1\right] \sin ^{2} \psi
$$

with

$$
\zeta=K_{q}^{\prime} L^{* 2}-K_{s_{0}} \eta_{x, s_{0}} \kappa_{s_{0}}
$$

where $\zeta$ characterizes the chromaticity compensation and $\kappa_{s_{0}}=\beta_{s_{0}} \beta_{0}^{*}$. Moreover, the $\beta$ function at the FD quadrupole $\left(\beta_{\mathrm{FD}}\right)$ can be expressed as

$$
\begin{aligned}
\frac{\beta_{\mathrm{FD}}(\delta)}{\beta_{0}^{*}}= & K_{s_{0}}^{2} \eta_{x, s_{0}}^{2} \beta_{s_{0}}^{2}(\xi \cos \psi-\sin \psi)^{2} \delta^{2} \\
& +2 K_{s_{0}} \eta_{x, s_{0}} \beta_{s_{0}}(\xi \cos \psi-\sin \psi)(\xi \sin \psi+\cos \psi) \delta \\
& +\xi^{2}+1
\end{aligned}
$$

which is proportional to $K_{s_{0}} \eta_{x, s_{0}} \beta_{s_{0}}(\xi \cos \psi-\sin \psi)$ and becomes extremely wide for $\psi \approx\left(n+\frac{1}{2}\right) \pi$.

Let us consider a "minimal" configuration of the localstyle FFS without the upstream bend, i.e., $\left(\eta_{x, s_{0}}=0\right)$. The paired sextupole at $s_{0}$ is solely for canceling geometric aberrations. The expression of chromatic $\beta^{*}$ can be simplified as

$$
\frac{\beta^{*}(\delta)}{\beta_{0}^{*}}=K_{q}^{\prime 2} L^{* 2} \delta^{2} \xi^{2}+\left(K_{q}^{\prime} L^{*} \delta+1\right)^{2}
$$

which eases the restriction on the phase advance between the two sextupoles for chromaticity correction. Besides, the chromatic variation of the $\beta_{\mathrm{FD}}$ vanishes.

The above derivations are also valid for the traditionalstyle FFS by muting the sextupole at $s_{1}$ and the bend between the sextupoles, i.e., $K_{s_{1}}=0, \eta_{x, s_{1}}=0$. The sextupole at $s_{0}$ represents chromaticity correction by means of two paired sextupoles with $-I$ transformation in between. Hence, $K_{q}^{\prime}$ in the Eq. (3)-(8) should be replaced by $K_{q}$. With satisfactory chromaticity correction, comparable IP bandwidths are expected for the two schemes, but the FD bandwidth might be wider for the local scheme, as 
predicted in Ref. [2]. A larger FD bandwidth is favored for reducing beam loss around the FD and relaxing requirement on the collimation system. These analytical estimations show the chromatic $\beta$ functions at the IP and FD, as an indication of the momentum bandwidths in terms of beam size and luminosity. The real momentum bandwidth also depends on higher-order chromatic terms and chromatic attributes of incoming beam. For an accurate evaluation of momentum bandwidth, tracking simulation in the presence of a realistic optic model, high-order magnetic components and machine imperfections will be necessary.

\section{SIMULATION}

To evaluate the momentum bandwidth of ATF2, a script including the IP beam size tuning procedure in the presence of static machine imperfections has been developed based on SAD [37], a computer program complex for accelerator design developed at KEK. Details of the incorporated static imperfections and the tuning procedures are described in Ref. [26]. Besides, the measured high-order components of FFS magnets have been considered [38]. For each study, more than 100 machines are simulated to pursue sufficient statistics. The Twiss parameters are computed with transfer matrix and the IP beam size is determined by Gaussian fitting of beam core. The simulation uses two optics that have been recently tuned at ATF2, the $10 \beta_{x}^{*} 1 \beta_{y}^{*}$ optics and the ultralow $\beta^{*}$ optics. To compare to measurements, we have taken into account a larger IP vertical beam size matching experimental observations and the chromatic optical parameters at the beam extraction.

\section{A. Design optics}

The momentum bandwidths in terms of IP $\beta$ functions and beam sizes are evaluated for on-momentum IP vertical beam sizes $\left(\sigma_{y 0}^{*}\right)$ of $36.3 \pm 2.6$ and $20.5 \pm 1.8 \mathrm{~nm}$, for the $10 \beta_{x}^{*} 1 \beta_{y}^{*}$ optics and ultralow $\beta^{*}$ optics, respectively, as shown in Fig. 3. Notice that a momentum spread of $0.08 \%$ is used for beam size evaluations. In general, the horizontal bandwidths in terms of beam size are much wider than the vertical bandwidths for the two optics. The horizontal bandwidths in terms of $\beta$ function and beam size appear quartic functions with a region where $\beta_{x}^{*}$ and $\sigma_{x}^{*}$ are smaller than the on-momentum values. Behaviors of chromatic horizontal $\beta$ functions and beam sizes are different for the ultralow $\beta^{*}$ optics, which could be interpreted by profile distortions, asymmetry and long tail, due to horizontal chromo-geometric aberrations. The vertical bandwidths of the $10 \beta_{x}^{*} 1 \beta_{y}^{*}$ optics have a region with smaller $\beta_{y}^{*}$ and $\sigma_{y}^{*}$ than on-momentum values. The ultralow $\beta^{*}$ optics presents narrower vertical bandwidths, that are shrunk by residual high-order chromatic aberrations, e.g., $U_{3236}$ and $U_{3246}$ where $U_{i j k l}=\partial^{3} x_{i} / \partial x_{j} \partial x_{k} \partial x_{l}$ and $x_{i}$ are the six dimension phase space coordinates. The vertical bands shown for the simulation results represent statistic errors over 100 machines. Here, the chromatic orbit at the extraction point with nonzero horizontal dispersion has been considered. Assuming a beam-energy jitter of $\pm 0.1 \%$ (uniformly distributed), the IP vertical single-shot beam size increase by $0.5 \pm 4.1 \%$ and $3.8 \pm 7.5 \%$ for the $10 \beta_{x}^{*} 1 \beta_{y}^{*}$ and ultralow $\beta^{*}$ optics, respectively.

The 10\% momentum bandwidths for the two optics are summarized in Table II in comparison with Next Linear Collider (NLC), ILC and CLIC. For the NLC FFS, the $10 \%$ momentum bandwidth for either traditional or local schemes is above $1 \%$ thanks to the supplemental improvements using nonlinear elements. The momentum bandwidths of ILC and ILC FFS are particularly optimized regarding the luminosity performance and may require further optimizations with nonlinear elements. The ultralow $\beta^{*}$ optics of ATF2 presents the smallest $10 \%$ momentum bandwidths because of its higher chromaticity. Besides, $\beta$ function at the beam waist $\left(\beta_{w}\right)$, i.e., the minimum $\beta$-function around the IP, can be inferred from Twiss parameters at the IP and experimentally determined through FD quadrupole scan. The evaluations show wide regions with smaller $\beta_{w}$ than the on-momentum value for both $10 \beta_{x}^{*} 1 \beta_{y}^{*}$ and ultralow $\beta^{*}$ optics (see Fig. 3). Notice that the estimated horizontal $\beta_{w}$ of the ultralow $\beta^{*}$ optics might be unpractical for large momentum deviations owing to strong horizontal chromatic aberrations.

Another important chromatic property is the IP beamorbit offset that can cause significant luminosity degradation and shrink measured momentum bandwidth. In the case of ATF2, a small IP orbit offset will perturb the interaction between the electron beam and IPBSM interference pattern and enlarge observed beam size. The IP beam positions displace by $10 \%$ of on-momentum beam sizes with beam energy deviations of about $0.13 \%$ and $0.07 \%$, for the $10 \beta_{x}^{*} 1 \beta_{y}^{*}$ and ultralow $\beta^{*}$ optics, respectively, as shown in Fig. 4. Assuming a beam-energy jitter of $0.1 \%$, an extra IP vertical position jitter of less than $5 \mathrm{~nm}$, four times smaller than the expected betatron beam position jitter $(\sim 20 \mathrm{~nm})$, is predicted for the two optics. For a large beam-energy offset of more than $\pm 0.5 \%$, the horizontal orbit displacement approximates the IPBSM laser spot size $(\sim 20 \mu \mathrm{m})$, and mismatches the laser-beam overlap. Therefore, one must optimize the transverse laser-beam overlap at every beam energy for the momentumbandwidth measurement at ATF2.

\section{B. Toward measurement}

Although an IP vertical beam size of $60 \mathrm{~nm}$ and below has been experimentally achieved in both $10 \beta_{x}^{*} 1 \beta_{y}^{*}$ and ultralow $\beta^{*}$ optics, tens of nanometers of reduction is still required to reach the design beam sizes. Furthermore, successful tuning of the IP vertical beam size is always challenging and could hardly be guaranteed for every operation period. The final IP vertical beam size is usually limited by the residual chromaticity, aberrations and 

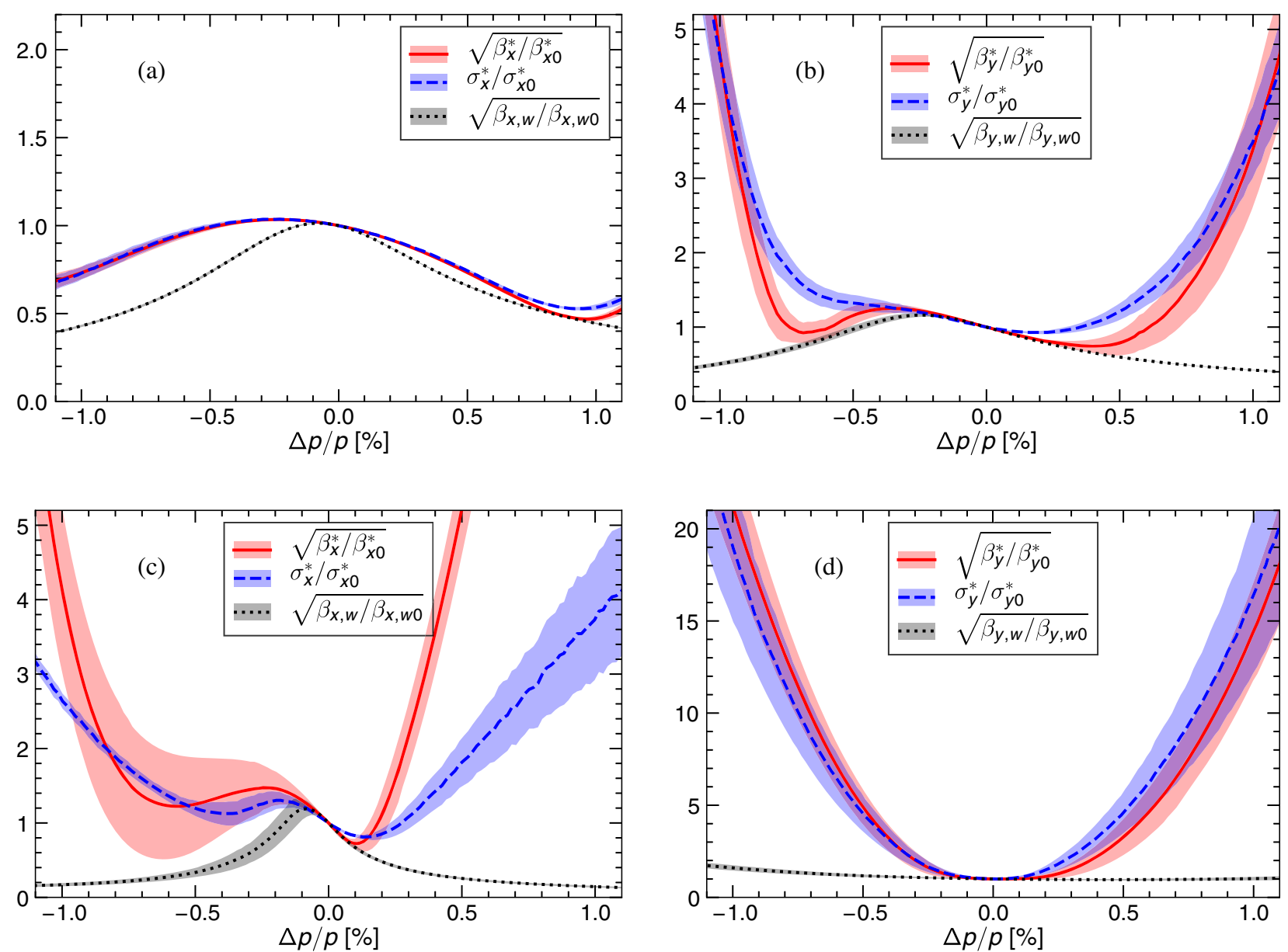

FIG. 3. Momentum bandwidths of the ATF2 $10 \beta_{x}^{*} 1 \beta_{y}^{*}$ optics (a, b) and ultralow $\beta^{*}$ optics (c, d). $\sigma_{x}^{*}$ and $\sigma_{y}^{*}$ are the IP horizontal and vertical beam sizes, respectively. $\beta_{x, w}$ and $\beta_{y, w}$ are the horizontal and vertical $\beta$ functions at beam waist, respectively. The actual random momentum offset is roughly the momentum spread $(0.08 \%)$ and the resulted beam-size growth is small comparing with other systematic effects.

high-order dispersions, that should be corrected with dedicated tuning knobs. Therefore, we have evaluated the momentum bandwidths for a larger on-momentum IP vertical beam size up to $\sim 100 \mathrm{~nm}$ at different stages of the tuning procedure. For the $10 \beta_{x}^{*} 1 \beta_{y}^{*}$ optics, $\sigma_{y 0}^{*}$ can be reduced to $37.7 \pm 0.8 \mathrm{~nm}$ with a few iterations of linear- knob corrections, but the vertical momentum bandwidth is worse than that with subsequent careful corrections of second-order chromaticity and aberrations, reaching a $\sigma_{y 0}^{*}$ of $36.3 \pm 0.3 \mathrm{~nm}$, as shown in Fig. 5(a). For a $\sigma_{y 0}^{*}$ of $59.5 \pm 5.5 \mathrm{~nm}$, that contains more residual linear aberrations, the vertical momentum bandwidth appears broader.

TABLE II. Momentum bandwidths for the ATF2 $10 \beta_{x}^{*} 1 \beta_{y}^{*}$ and ultralow $\beta^{*}$ optics, in comparison with the NLC, ILC, and CLIC baseline designs $[1,10,14,39] . \xi_{y}$ is the vertical chromaticity, $\gamma \varepsilon_{y}$ the normalized vertical emittance, $\sigma_{\delta}$ the rms momentum spread and $\chi_{m}$ the $10 \%$ momentum bandwidth. All the assessments employ error-free lattices without synchrotron radiation.

\begin{tabular}{lccccccc}
\hline \hline & $L^{*}[\mathrm{~m}]$ & $\beta_{y}^{*}[\mathrm{~mm}]$ & $\xi_{y}\left[10^{4}\right]$ & $\gamma \varepsilon_{y}[\mathrm{~nm} . \mathrm{rad}]$ & $\sigma_{y, \text { design }}^{*}[\mathrm{~nm}]$ & $\sigma_{\delta}$ \\
\hline NLC $(500 \mathrm{GeV})$ & 2 & $(0.125 / 0.12)^{\mathrm{a}}$ & $(1.67 / 1.6)^{\mathrm{a}}$ & $(70 / 35)^{\mathrm{a}}$ & $(4.2 / 2.0)^{\mathrm{a}}$ & $0.3 \%$ & $(1.22 \% / 1.33 \%)^{\mathrm{a}}$ \\
ILC $(250 \mathrm{GeV})$ & 4.1 & 0.41 & 1.0 & 35 & 7.6 & $0.2 \%$ & $0.57 \%$ \\
ILC $(500 \mathrm{GeV})$ & 4.5 & 0.48 & 0.93 & 35 & 5.9 & $0.124 \%$ & $0.75 \%$ \\
CLIC $(380 \mathrm{GeV})$ & 6 & 0.1 & 6.0 & 30 & 2.3 & $0.3 \%$ & $0.54 \%$ \\
CLIC $(3 \mathrm{TeV})$ & 6 & 0.120 & 5.0 & 20 & 0.9 & $0.3 \%$ & $0.35 \%$ \\
ATF2 $10 \beta_{x}^{*} 1 \beta_{y}^{*}$ & 1 & 0.1 & 1.0 & 30 & 37 & $0.08 \%$ & $0.64 \%$ \\
ATF2 ultralow $\beta^{*}$ & 1 & 0.025 & 4.0 & 30 & 23 & $0.08 \%$ & $0.31 \%$ \\
\hline \hline
\end{tabular}

${ }^{\mathrm{a}}$ For traditional/local schemes. 

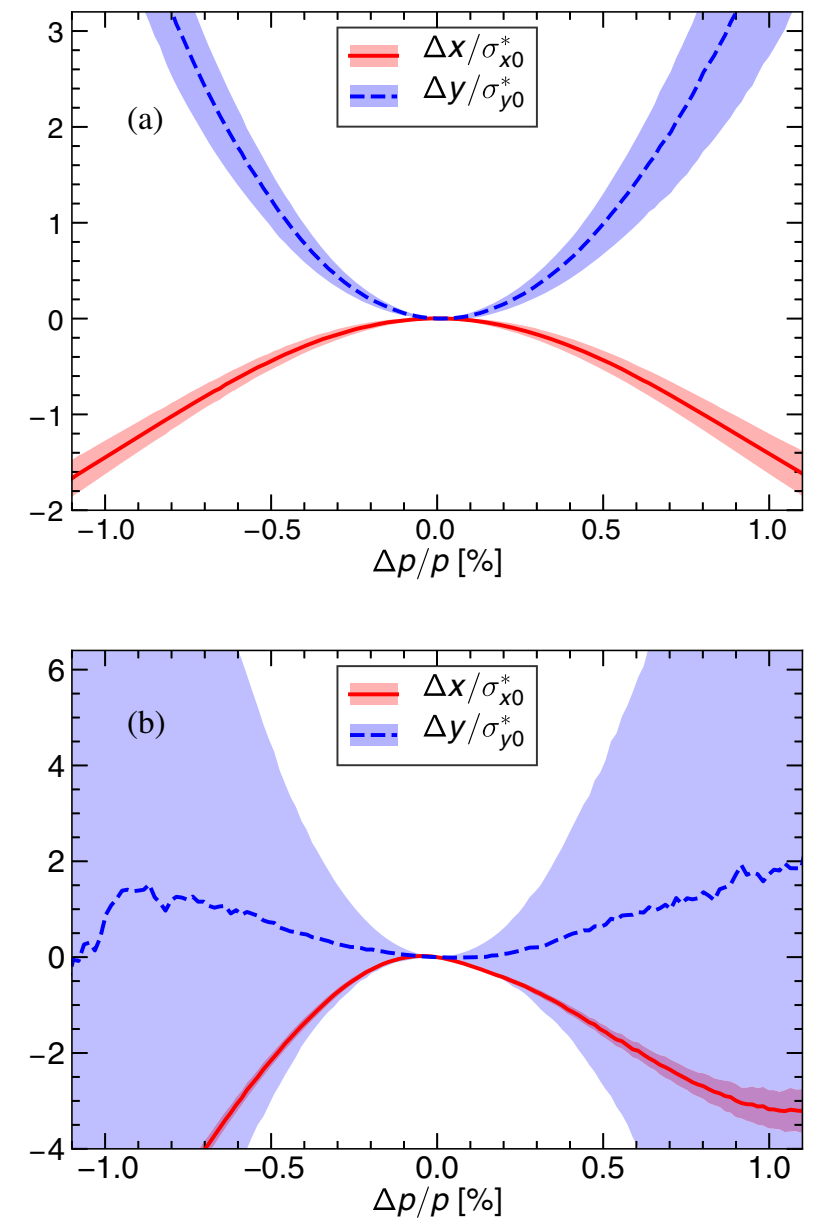

FIG. 4. Transverse IP beam-position displacements for the $10 \beta_{x}^{*} 1 \beta_{y}^{*}$ optics (a) and ultralow $\beta^{*}$ (b) optics.

For the ultralow $\beta^{*}$ optics, $\sigma_{y 0}^{*}$ decreases to $76.2 \pm 1.5 \mathrm{~nm}$ after linear-aberration corrections, and the vertical momentum bandwidth is much broader than that with complete tuning-knob corrections, obtaining a $\sigma_{y 0}^{*}$ of $25.5 \pm 3.3 \mathrm{~nm}$, as shown in Fig. 5(b). This is thought to be owing to the notable residual aberrations in the absence of nonlinear knob corrections. With further corrections of second-order terms, the vertical momentum bandwidth becomes narrower and closer to the design, especially after obtaining a $\sigma_{y 0}^{*}$ of $39.2 \pm 2.1 \mathrm{~nm}$ with one iteration of nonlinear tuning knobs. Moreover, the horizontal bandwidth is insensitive to the increase of IP vertical beam size $(<0.5 \mu \mathrm{m})$. Regardless, a successful tuning of the onmomentum IP beam size will be crucial for vertical momentum-bandwidth measurement. Besides, a similar on-momentum beam size should be matched for the latter comparison of measurement and simulation.

For the momentum bandwidth measurements at ATF2, beam centroid energy is shifted via the rf frequency ramp in the DR. Subsequently, the chromatic optics deviations of the DR might further shrink the momentum-bandwidth observations. To evaluate its impact, we have measured the
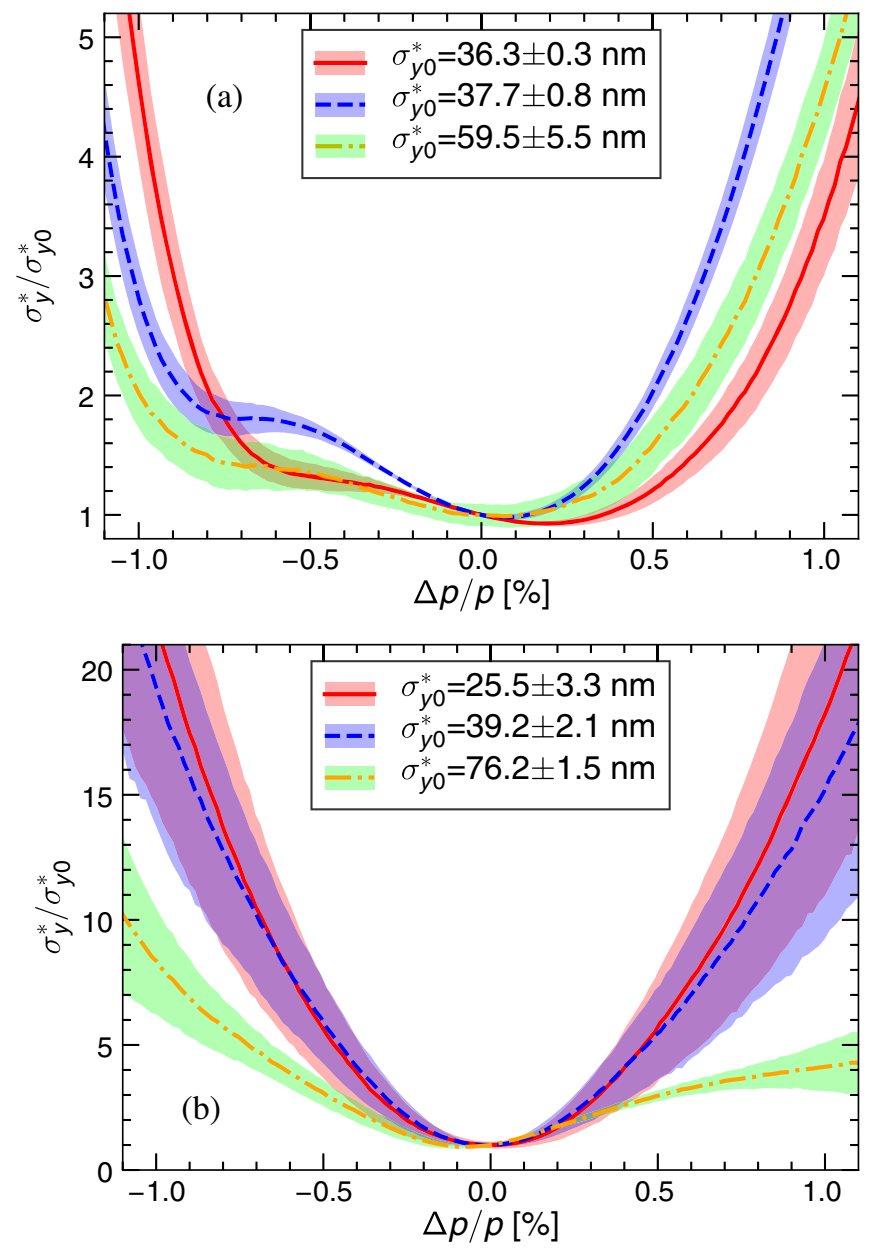

FIG. 5. Momentum bandwidths versus on-momentum IP vertical beam size for the $10 \beta_{x}^{*} 1 \beta_{y}^{*}$ optics (a) and ultralow $\beta^{*}$ optics (b). The on-momentum beam sizes of $36.3 \pm 0.3,37.7 \pm 0.8$ and $59.5 \pm$ $5.5 \mathrm{~nm}$ in (a) are obtained with careful linear and nonlinear knobs corrections, linear knob corrections, and preliminary linear knob corrections (one iteration), respectively. The on-momentum beam sizes of $25.5 \pm 3.3,39.2 \pm 2.1$ and $76.2 \pm 1.5 \mathrm{~nm}$ in (b) are achieved after applying complete linear and nonlinear knobs corrections, linear knob corrections plus one iteration of nonlinear knobs, and linear knob corrections, respectively.

chromatic Twiss parameters and vertical emittance at the extraction point, as shown in Fig. 6 (a-c). The Twiss parameters are inferred from the K-modulation measurements at the neighboring quadrupoles. The vertical emittance is measured using an X-ray synchrotron radiation (XSR) monitor $[40,41]$. The vertical $\beta$ function at the source point is extrapolated by fitting the $\beta$ functions measured at five adjacent quadrupoles. However, the horizontal emittance is not simultaneously acquired due to a lack of accurate horizontal-dispersion measurement at the source point. Alternatively, we have assessed the chromatic horizontal emittance and dispersions at the extraction through simulation, as shown in Fig. 6 (d-f). In the simulation, the operational vertical emittance is approached by introducing random rolling error to the 

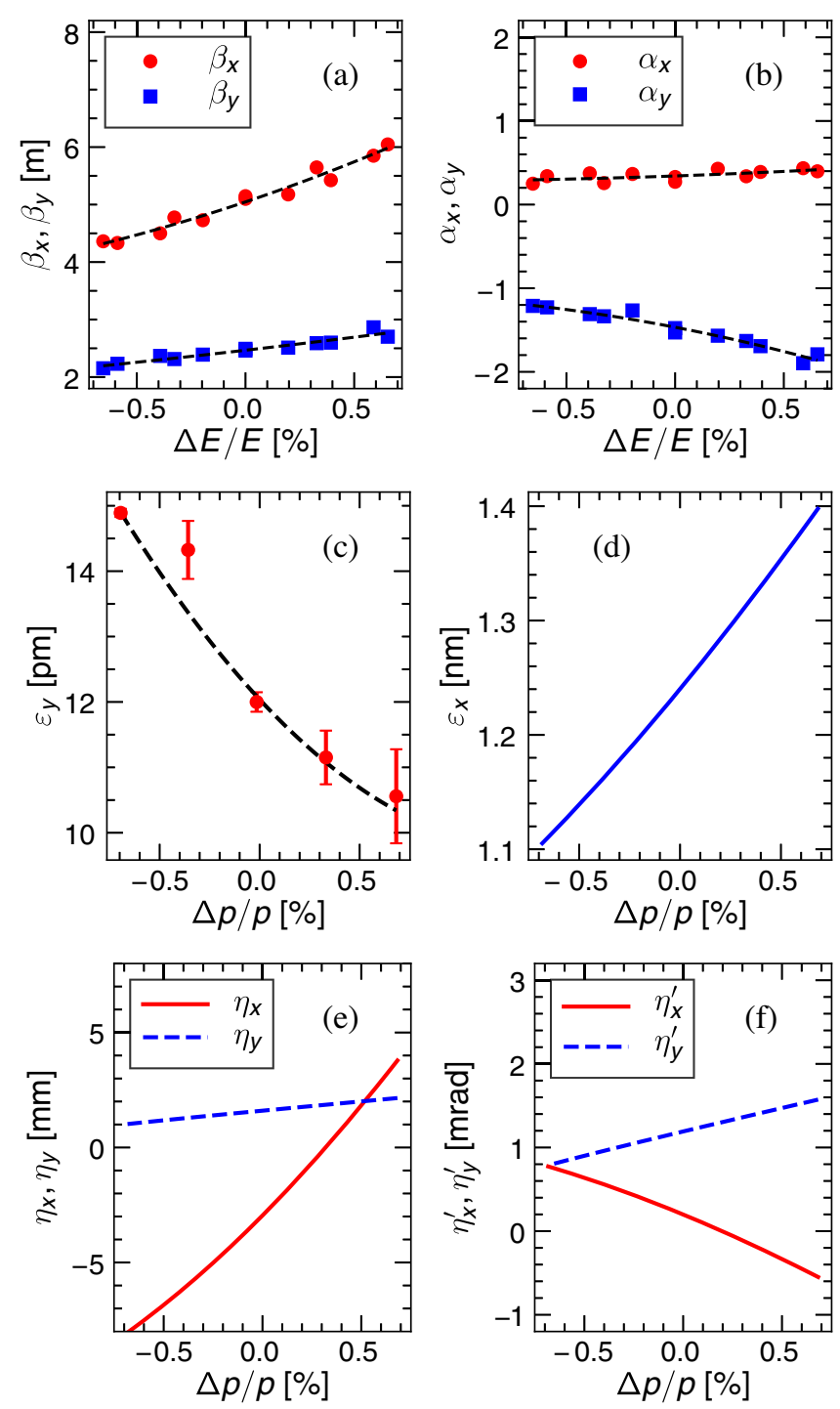

FIG. 6. Chromatic Twiss parameters (a, b), emittances (c, d) and dispersion functions (e, f) at the extraction. The Twiss parameters and vertical emittance are from measurement while the others are predicted through simulation. The dashed lines in $(\mathrm{a}-\mathrm{c})$ are fits to the experimental data.

quadrupoles in the straight sections and dispersion bump using selected steering magnets [42], as depicted in Fig. 7. We have only reevaluated the momentum bandwidths of the ultralow $\beta^{*}$ optics for an on-momentum IP vertical beam size of less than $30 \mathrm{~nm}$. Including the chromatic beam-parameter deviations at the extraction, the vertical momentum bandwidth tends to be narrower, as shown in Fig. 8. The difference between horizontal bandwidths with a momentum offset of $0.5-1.3 \%$ can be explained by the chromatic horizontal dispersion at the extraction point. For bandwidth measurements in a small momentum range, e.g., within $[-1 \%, 0.6 \%]$, knowledge of chromatic beam parameters at the extraction is favorable but might not be essential.

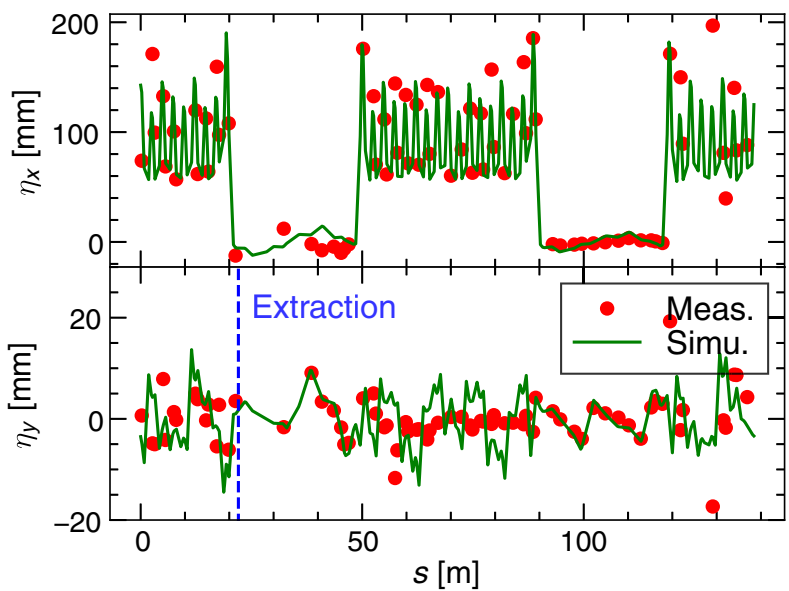

FIG. 7. Measurement and reproduction of horizontal and vertical dispersions of the ATF DR. The equilibrium vertical emittance is about $12 \mathrm{pm}$ in the presence of intra-beam scattering with a bunch charge of $0.16 \mathrm{nC}$.

\section{MEASUREMENT}

The vertical momentum bandwidth has been measured in the $10 \beta_{x}^{*} 1 \beta_{y}^{*}$ and ultralow $\beta^{*}$ optics, for a stable vertical beam size of less than $70 \mathrm{~nm}$. Meanwhile, the horizontal momentum bandwidth has been merely measured in ultralow $\beta^{*}$ optics with an IP vertical beam size of about $150 \mathrm{~nm}$. The rf-frequency ramp is limited to $\pm 10 \mathrm{kHz}$ around the central frequency $(714 \mathrm{MHz})$, corresponding to a momentum offset within $\pm 0.68 \%$. The linearity of momentum offset as a function of rf frequency modulation has been verified, as shown in Fig. 9. The relative momentum deviation is inferred from the orbit changes in the arc section.



FIG. 8. Momentum bandwidths with fixed and chromatic Twiss parameters, emittances, and dispersions at the extraction for the ultralow $\beta^{*}$ optics. The on-momentum IP vertical beam sizes are $27.5 \pm 3.2$ and $27.4 \pm 3.1 \mathrm{~nm}$ for the two cases. 


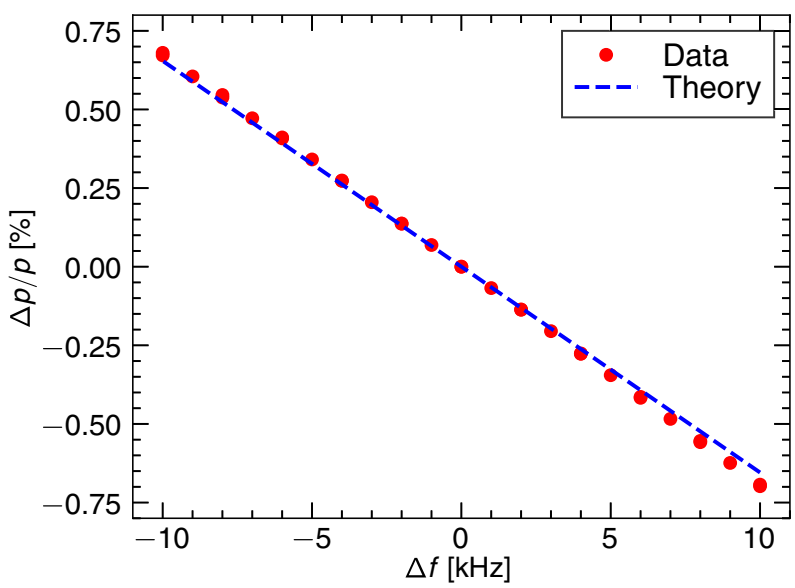

FIG. 9. Momentum offset as a function of $\mathrm{rf}$ frequency modulation. The maximum discrepancy between theoretical predictions and measurements is about $4 \times 10^{-4}$.

The IP vertical beam size is measured employing the IPBSM. The electron beam is scanned across the interference fringe pattern, formed by two laser paths, to yield a modulated rate of Compton-scattered photons in the forward direction. The vertical beam size is extrapolated from the modulation depth, which is normally overestimated due to systematic errors, e.g., beam jitter, wakefield effect and modulation reduction due to deformation of interference fringe. Embracing these systematic effects, the measured beam size $\left(\sigma_{y, \text { IPBSM }}\right)$ and the single-shot beam size can be correlated by

$$
\sigma_{y, \mathrm{IPBSM}}=\frac{1}{2 k_{y}} \sqrt{2\left(2 k_{y}^{2} \sigma_{y, \mathrm{~m}}^{2}-\log C\right)}
$$

with

$$
\sigma_{y, \mathrm{~m}}^{2}=\sigma_{y 0}^{2}+\sigma_{\Delta y}^{2}+\sigma_{w}^{2}
$$

where $k_{y}=2 \pi \sin (\theta / 2) / \lambda, \theta$ the crossing angle, $\lambda$ the wavelength, $\sigma_{y, \mathrm{~m}}$ the multishot beam size, $\sigma_{y, 0}$ the single-shot beam size, $\sigma_{\Delta y}$ the beam position jitter, $\sigma_{w}$ the beam size growth from wakefields, and $C$ the modulation reduction due to deformed interference fringe. In consequence, the single-shot vertical beam size can be extrapolated from measurements regarding the preceding evaluations of these systematic effects [26], as summarized in Table III.

In the $10 \beta_{x}^{*} 1 \beta_{y}^{*}$ optics, the vertical momentum bandwidth has been measured with an on-momentum IP beam size of $57.4 \pm 1.8 \mathrm{~nm}$ (measured value), as shown in Fig. 10(a). The measurements show an asymmetric shape with a minimum beam size of $56.0 \pm 1.9 \mathrm{~nm}$ at a momentum offset of $0.07 \%$. The IP vertical beam size increases by $52 \pm 10 \%$ and $90 \pm 14 \%$ for the maximum momentum deviations of $\pm 0.39 \%$. Comparing to the simulation
TABLE III. Major systematic errors for the IP beam size measurements in the IPBSM $174^{\circ}$ mode with a low bunch charge $(0.16 \mathrm{nC})$. The wakefield induced beam size growths are determined experimentally in the same operation weeks as the momentum bandwidth measurements. The IP position jitter and IPBSM modulation reduction factor are from analytical assessments concerning realistic machine parameters. Notice that these systematic errors closely depend on machine conditions and vary from one operation period to another.

\begin{tabular}{lccc}
\hline \hline Optics & $\sigma_{\Delta y}[\mathrm{~nm}]$ & $\sigma_{w}[\mathrm{~nm}]$ & $C$ \\
\hline $10 \beta_{x}^{*} 1 \beta_{y}^{*}$ & 22.6 & 24.2 & 0.94 \\
ultralow $\beta^{*}$ & 20.8 & 20.0 & 0.91 \\
\hline \hline
\end{tabular}

predictions using the measured Twiss parameters at the extraction, the measurements show stronger momentum dependence, mainly at lower beam energy. The vertical single-shot beam sizes at nominal beam energy are
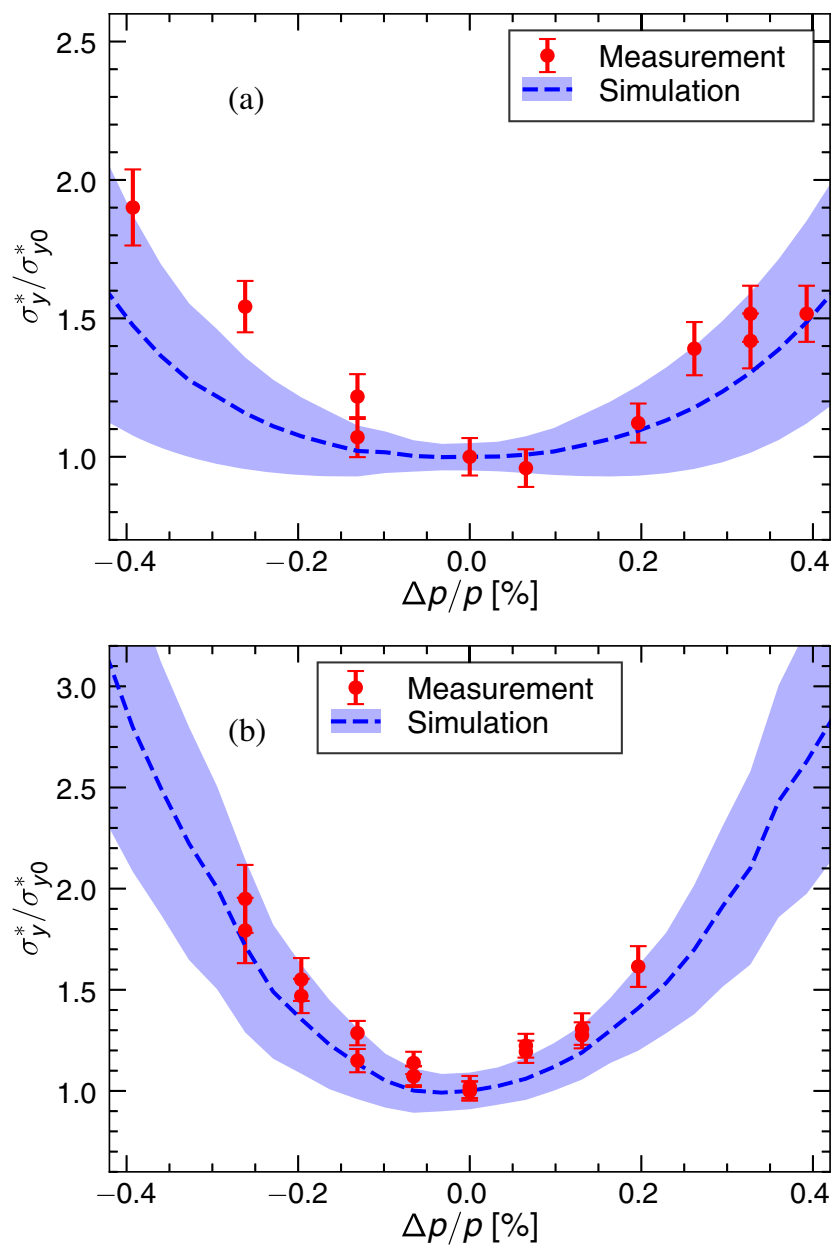

FIG. 10. The measured and simulated vertical momentum bandwidths for the $10 \beta_{x}^{*} 1 \beta_{y}^{*}$ (a) and ultralow $\beta^{*}$ (b) optics. The beam sizes measured by the IPBSM have been converted to single-shot beam sizes with respect to the systemtatic errors in Table III. 
$44.6 \pm 2.4$ and $44.6 \pm 1.6 \mathrm{~nm}$ for the measurements and simulations, respectively.

In the ultralow $\beta^{*}$ optics, the vertical momentum bandwidth has been measured for a momentum deviation within $[-0.26 \%, 0.2 \%]$, limited by the measuring range of the IPBSM $174^{\circ}$ mode. The on-momentum IP vertical beam size is $62.9 \pm 1.6 \mathrm{~nm}$, corresponding to a single-shot beam size of $52.7 \pm 1.9 \mathrm{~nm}$. As shown in Fig. 10(b), the observed bandwidth is narrower than that of the $10 \beta_{x}^{*} 1 \beta_{y}^{*}$ optics: the vertical IP beam size increases by about $90 \%$ for a momentum deviation of $-0.26 \%$. Moreover, the observation is slightly narrower than the simulation, which has an on-momentum IP beam size of $49.4 \pm 3.2 \mathrm{~nm}$, probably because of unexpected high-order chromatic aberrations, imprecise optics model for simulation reproduction, and other IPBSM diagnostic errors.

The horizontal momentum bandwidth and the chromatic horizontal $\beta$ function at the waist have been measured in the ultralow $\beta^{*}$ optics for an on-momentum IP horizontal beam size of about $11 \mu \mathrm{m}$ and a momentum deviation in $[-0.4 \%$, $0.64 \%]$. The IP horizontal beam size is measured
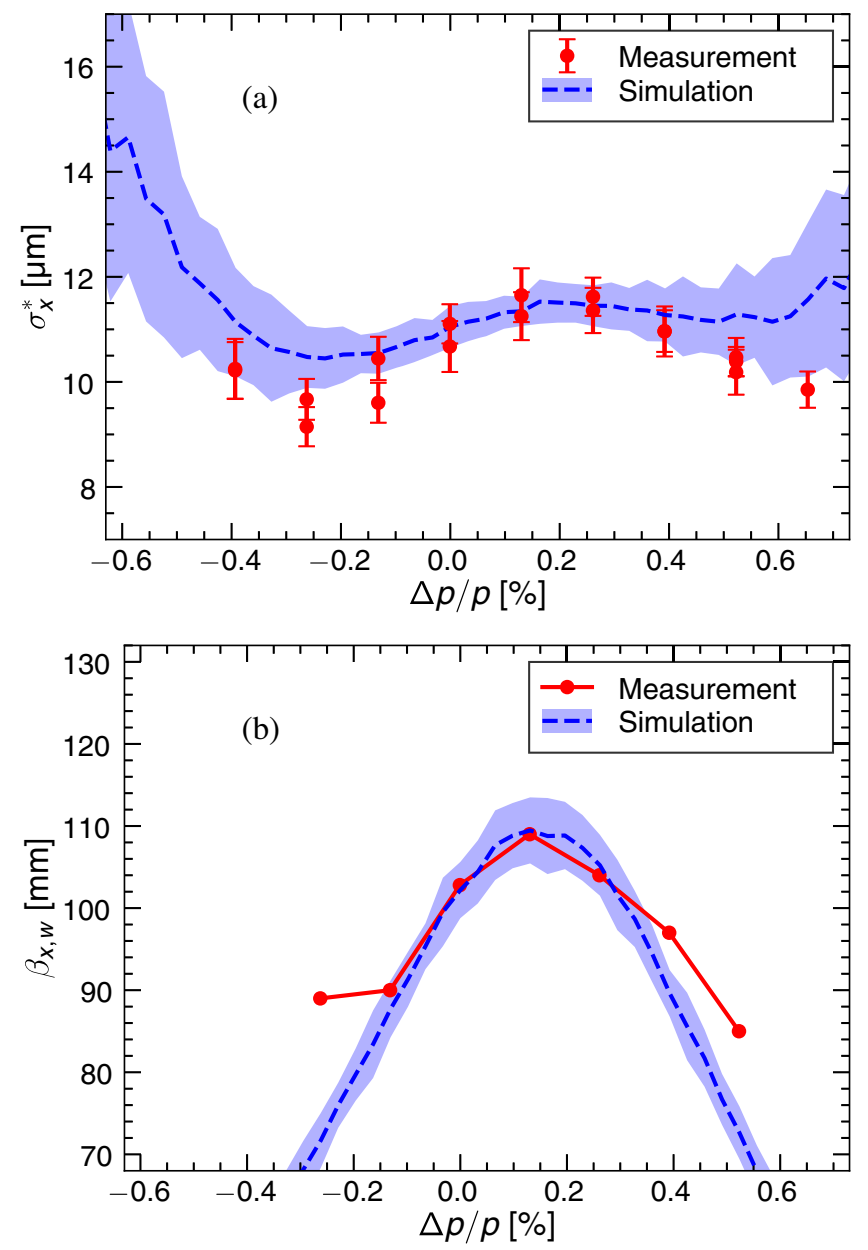

FIG. 11. Measurements of the horizontal momentum bandwidth (a) and chromatic horizontal $\beta$ function at the waist (b) in the ultralow $\beta^{*}$ optics. employing a wire scanner and the $\beta$ function at the waist is determined with quadrupole-scan method. The offmomentum horizontal beam size is smaller than the onmomentum value for momentum deviations of less than zero and higher than $0.39 \%$, as shown in Fig. 11(a). Moreover, the observation has the same trend as the simulation prediction, that has a vertical IP beam size of $123.7 \pm 13.7 \mathrm{~nm}$. The horizontal $\beta$ function at the waist decreases versus the momentum offset, consistent with the simulations, as shown in Fig. 11(b). Notice that the measurements have been deliberately shifted by $+0.13 \%$ to compensate the sudden drift of the rf cavity's analog control voltage at the beginning of measurements.

\section{CONCLUSION}

As an important FFS characteristic, the momentum bandwidth has been studied numerically and experimentally for the ATF2 case. Analytical expressions for assessing the chromatic $\beta$ function at the IP and FD have been derived concerning a simplified FFS model. These formulas are appropriate to describe some basic chromatic properties of both traditional and local final-focus schemes. Simulations in the presence of static machine errors show wide and quartic like horizontal bandwidth and relatively narrower vertical bandwidth in a parabola shape. A momentum region, where the horizontal IP beam size is smaller than the on-momentum value, has been indicated. The $10 \%$ momentum bandwidths are evaluated as $0.64 \%$ and $0.31 \%$ for $10 \beta_{x}^{*} 1 \beta_{y}^{*}$ and ultralow $\beta^{*}$ optics, respectively. Chromatic IP beam-position offset, which induces additional beam jitter in the presence of beam-energy jitter and shrinks momentum bandwidth, has been predicted. Optimization of IPBSM laser-beam overlap at every beam energy has been suggested for momentum-bandwidth measurements at ATF2. Moreover, the possible impacts from an improperly tuned IP vertical beam size and the chromatic beam parameters at the extraction have been estimated. For the comparison with design, a small onmomentum vertical IP beam size is vital for the vertical momentum-bandwidth measurement, but not for the horizontal case. For a momentum deviation within $[-1 \%, 0.6 \%]$, minor deformation of the momentum bandwidth from chromatic incoming beam has been verified.

The measured vertical momentum bandwidths are slightly narrower than the simulation predictions for the $10 \beta_{x}^{*} 1 \beta_{y}^{*}$ and ultralow $\beta^{*}$ optics, probably owing to unknown residual high-order aberrations and diagnostic errors. The horizontal momentum bandwidth measured in the ultralow $\beta^{*}$ optics agrees well with simulation and shows two regions with smaller IP horizontal beam sizes. Besides, decrease of the horizontal $\beta$ function at the waist versus momentum offset has also been observed. For the near future, simultaneous measurements of the vertical and horizontal momentum bandwidths and optimizations of the ultralow $\beta^{*}$ optics toward a wider momentum bandwidth 
have been proposed. Further experimental investigations, along with the reduction of vertical IP beam size, are strongly recommended in preparation of the FLC. In addition, assessments of high-order aberrations using "stepwise ray-tracing" tools would be also interesting when the realistic field maps are available.

\section{ACKNOWLEDGMENTS}

The authors would like to express their gratitude to the ATF2 collaboration and the staff of ATF. Special thanks go to P. Bambade, K. Oide and G. White for very useful discussions and suggestions in simulations.

[1] C. Adolphsen, R. Aiello, R. Alley, R. Assmann, K. Bane, T. Barklow et al., Zeroth-order design report for the Next Linear Collider, Report No. SLAC-R-474, 1996.

[2] P. Raimondi and A. Seryi, Novel Final Focus Design for Future Linear Colliders, Phys. Rev. Lett. 86, 3779 (2001).

[3] T. Raubenheimer and F. Zimmermann, Final-focus systems in linear colliders, Rev. Mod. Phys. 72, 95 (2000).

[4] H. G. Morales and R. Tomás, Final-focus systems for multi-TeV linear colliders, Phys. Rev. Accel. Beams 17, 101001 (2014).

[5] ILC Global Design Effort, ILC Technical Design Report, 2013.

[6] P. Bambade, T. Barklow, T. Behnke, M. Berggren, J. Brau, P. Burrows et al., The International Linear Collider: A Global Project, arXiv:1903.01629.

[7] M. Aicheler, P. Burrows, M. Draper, T. Garvey, P. Lebrun, K. Peach et al., CLIC Conceptual Design Report, 2014.

[8] R. Tomás, Overview of the Compact Linear Collider, Phys. Rev. Accel. Beams 13, 014801 (2010).

[9] A. Seryi, R. Tomás, F. Zimmermann, K. Kubo, S. Kuroda, T. Okugi, T. Tauchi, N. Terunuma, J. Urakawa, G. White, M. Woodley, and D. Angal-Kalinine, Experimental and theoretical progress of linear collider final focus design and ATF2 facility, Nucl. Instrum. Methods Phys. Res., Sect. A 740, 2 (2014).

[10] P. Raimondi, A. Seryi, and P. Tenenbaum, Tunability of the NLC final focus system, in Proceedings of PAC01, Chicago, USA (IEEE, New York, 2001), pp. 3831-3833.

[11] R. Brinkmann, Optimization of a final focus system for large mommentum bandwidth, DESY Report No. M-9014, 1990.

[12] K. Oide, Final focus system with odd-dispersion scheme, SLAC-AP-KEK Preprint 92-58, 1992.

[13] R. Tomás, Nonlinear optimization of beam lines, Phys. Rev. Accel. Beams 9, 081001 (2006).

[14] F. Plassard, A. Latina, E. Marin, R. Tomás, and P. Bambade, Quadrupole-free detector optics design for the Compact Linear Collider final focus system at $3 \mathrm{TeV}$, Phys. Rev. Accel. Beams 21, 011002 (2018).

[15] A. Pastushenko, Nonlinear optimization of the ultra-low beta* optics, International Workshop on Future Linear Collider, Sendai, Japan (2019), https://agenda .linearcollider.org/event/8217/contributions/44511/
attachments/34958/54033/LCWS_2019_ATF2_oct_29_ 10_2019_2.pdf.

[16] M. Bermdt, Final Focus Test Beam Design Report, Report No. SLAC-REP-376, 1991.

[17] V. Balakin, V. Alexandrov, A. Mikhailichenko, K. Flöttmann, F. Peters, G. Voss et al., Focusing of Submicron Beams for TeV-Scale $e^{+} e^{-}$Linear Colliders, Phys. Rev. Lett. 74, 2479 (1995).

[18] P. Tenenbaum, Expanded studies of linear collider final focus systems at the final focus test beam, Report No. SLAC-R-95-475, 1995.

[19] V. Alexandrof, V. Balakin, A. Mikhailichenko, K. Flottmann, K. Peters, G. Voss et al., Results of final focus test beam, in Proceedings PAC95, Dallas, USA (IEEE, New York, 1995), pp. 2742-2746.

[20] K. Kubo, M. Akemoto, S. Anderson, T. Aoki, S. Araki, K. Bane et al., Extremely low vertical-emittance beam in the Accelerator Test Facility at KEK, Phys. Rev. Lett. 88, 194801 (2002).

[21] K. Honda, K. Kubo, S. Anderson, S. Araki, K. Bane, A. Brachmann et al., Achievement of ultralow emittance beam in the Accelerator Test Facility damping ring, Phys. Rev. Lett. 92, 054802 (2004).

[22] B. Grishanov, P. Logachev, F. Podgorny, V. Telnov, D. AngalKalinin, R. Appleby et al., ATF2 proposal (2005).

[23] G. White, R. Ainsworth, T. Akagi, J. Alabau-Gonzalvo, D. Angal-Kalinin, S. Araki et al., Experimental validation of a novel compact focusing scheme for future energy-frontier linear lepton colliders, Phys. Rev. Lett. 112, 034802 (2014).

[24] T. Okugi, Achievement of small beam size at ATF2 beamline, in Proceedings of LINAC2016, East Lansing, USA (JACOW, 2016), pp. 27-31, https://accelconf.web .cern.ch/linac2016/papers/mo3a02.pdf.

[25] M. Patecki, D. Bett, E. Marin, F. Plassard, R. Tomás, K. Kubo et al., Probing half $\beta_{y}^{*}$ optics in the Accelerator Test Facility 2, Phys. Rev. Accel. Beams 19, 101001 (2016).

[26] R. Yang, A. Pastushenko, A. Aryshev, M. Bergamaschi, V. Cilento, A. Faus-Golfe et al., Tuning the ultralow $\beta^{*}$ optics at the KEK Accelerator Test Facility 2, Phys. Rev. Accel. Beams 23, 071003 (2020).

[27] A. Aryshev, P. Bambade, D. Bett, L. Brunetti, P. Burrows, V. Cilento et al., ATF Report 2020, Report No. CERNACC-2020-0029, 2020.

[28] G. White, ATF2 optics design, ICFA Beam Dyn. Newslett. 61, 26 (2013), https://icfa-usa.jlab.org/archive/newsletter/ icfa_bd_nl_61.pdf.

[29] T. Okugi, S. Araki, P. Bambade, K. Kubo, S. Kurado, M. Masuzawa et al., Linear and second order optics corrections for the KEK Accelerator Test Facility final focus beam line, Phys. Rev. Accel. Beams 17, 023501 (2014).

[30] R. Tomás, H. Braun, J. Delahaye, E. Marin, D. Schulte, F. Zimmermann et al., ATF2 ultra-low $\beta_{y}^{*}$ proposal, in Proceedings of PAC09, Vancouver, Canada (IEEE, New York, 2009), pp. 1-3.

[31] E. Marin, R. Tomás, B. Parker, P. Bambade, S. Kuroda, T. Okugi et al., Scenarios for the ATF2 ultra-low betas proposal, in Proceedings of the International Particle Accelerator Conference, Kyoto, Japan (ICR, Kyoto, 2010), pp. 4554-4556. 
[32] E. Marin, R. Tomás, P. Bambade, K. Kubo, T. Okugi, T. Tauchi et al., Design and high order optimization of the Accelerator Test Facility lattices, Phys. Rev. Accel. Beams 17, 021002 (2014).

[33] T. Shintake, Proposal of a nanometer beam size monitor for e+e- linear colliders, Nucl. Instrum. Methods Phys. Res., Sect. A 311, 453 (1992).

[34] T. Suehara, M. Oroku, T. Yamanaka, H. Yoda, T. Nakamura, Y. Kamiya et al., A nanometer beam size monitor for ATF2, Nucl. Instrum. Methods Phys. Res., Sect. A 616, 1 (2010).

[35] J. Yan, Y. Yamaguchi, Y. Kamiya, S. Komamiya, M. Oroku, T. Okugi, N. Terunuma, K. Kubo, T. Tauchi, and J. Urakawa, Measurement of nanometer electron beam sizes with laser interference using Shintake monitor, Nucl. Instrum. Methods Phys. Res., Sect. A 740, 131 (2014).

[36] K. Oide, A final focus system for flat-beam linear colliders, Nucl. Instrum. Methods Phys. Res., Sect. A 276, 427 (1989).

[37] $\mathrm{SAD}$, is a computer program for accelerator design; see http://acc-physics.kek.jp/SAD/.
[38] M. Masuzawa, QEA Magnet Measurements at KEK and Comparison with IHEP Results, talk at the 11th ATF2 project meeting (2011).

[39] T. Okugi and V. Cilento (private communication).

[40] K. Iida, N. Nakamura, H. Sakai, K. Shinoe, H. Takaki, M. Fujisawa et al., Measurement of an electron-beam size with a beam profile monitor using Fresnel zone plates, Nucl. Instrum. Methods Phys. Res., Sect. A 506, 41 (2003).

[41] T. Naito, H. Hayano, K. Kubo, S. Kuroda, T. Okugi, N. Terunuma et al., Emittance measurements using a X-ray beam profile monitor at KEK-ATF, in Proceedings IBIC2012, Tsukuba, Japan (JACOW, 2012), pp. 215217, https://accelconf.web.cern.ch/IBIC2012/papers/mopb63 .pdf.

[42] K. Kubo, Simulation study of low emittance tuning of the Accelerator Test Facility damping ring at KEK, Phys. Rev. ST Accel. Beams 6, 092801 (2003). 